\title{
Dynamic stimulus display for the WGTA
}

\author{
PERRY M. NEALIS, ARMAND CARPENTIER, STEPHEN J. SUOMI, and HARRY F. HARLOW \\ University of Wisconsin, Madison, Wisconsin 59706
}

\begin{abstract}
A description is given of the design and construction of a three-stimulus display to be used in conjunction with a standard Wisconsin General Test Apparatus (WGTA). The display employs planometric stimuli that are presented by rear illumination of transparent images on closed loops of 70-mm photographic film. These stimuli can be set in motion to provide a stimulus dimension distinct from color, shape, pattern, size, and number. The results of a pilot experiment indicate that movement of test stimuli can be a potent stimulus cue in primate learning tasks.
\end{abstract}

Paradigms of primate learning have utilized test stimuli that represent a considerable variety of phrsical dimensions, e.g. color, size, form, brightness, and numerous combinations of these stimulus characteristics. In addition. discriminative stimuli. employed in certain sign-learning situations, constitute an equally diverse spectrum: simple pointers (Sayner \& Davis, 1972). variations of the color of the stimulus ground (Spaet \& Harlow. 1943), and the absolute size of the test stimuli (Warren. 1960). Although it has been shown that rhesus monkeys can learn to successfully discriminate direction of movement, e.g. upward vs. downward motion (Hamilton \& Lund. 1970), the efticacy of motion as a stimulus dimension in learning paradigms hats yet to be determined.

This paper describes a test apparatus that incorporates motion as a stimulus dimension in addition to color, shape, size, pattern, and number. The apparatus is designed to be used either in conjunction with a standard WGTA (Harlow, 1949) or in home-catge situations. A pilot experiment employing this apparatus and involving three-choice color and motion discrimination performance by three adult rhesis monkeys is also reported. The electronic hardware and circuitry are not discussed in detail because of their relative simplicity. A component listing and electrical diagram are available without charge upon written request to the authors.

\section{APPARATUS}

The stimulus display console, illustrated in Figure 1. consists of three stimulus windows with corresponding foodwells, as well as drive motors, lamp assemblies, reward delivery systems, and

This project was supported by USPHS Grant MH-11894 from the National Institute of Mental Health to the University of Wisconsin Primate Laboratory. The authors wish to thank Roy Curtis. Glen Lee, and Richard Pape for their technical assistance, and Hannah $W u$ for her help in carrying out the experiment. Reprint requests should be add ressed to Dr. Stephen J. Suomi, University of Wisconsin Primate Laboratory. 22 North Charter Street, Madison. Wisconsin 53706.

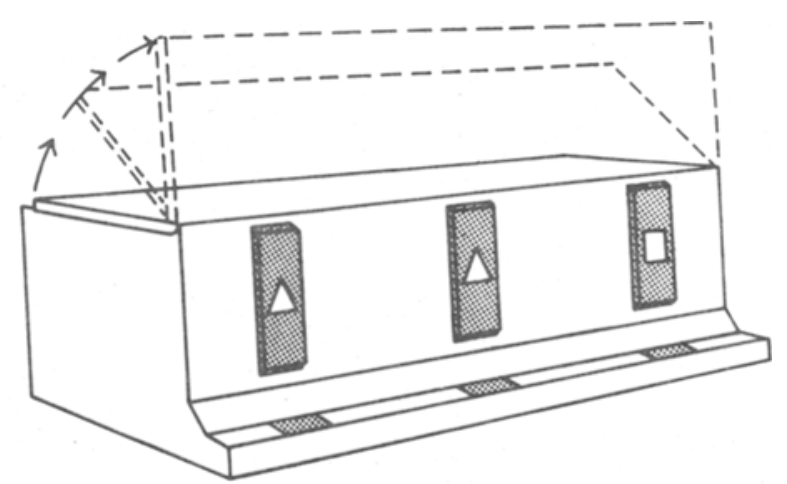

Figure 1. The stimulus display unit consists of three stimulus windows with corresponding foodwells. Stimuli are viewed through clear Plexiglas response manipulanda that overlay the windows. The dust cover rotates into a vertical position to obstruct the subject's view of the experimenter.

associated electrical circuitry. The frontispiece is $0.6-\mathrm{cm}$ flat-black Plexiglas with three $4.5 \times 13.5 \mathrm{~cm}$ transparent windows separated from center to center by $18 \mathrm{~cm}$. Each window is overlaid by a $0.6-\mathrm{cm}$-thick $5.5 \times 16 \mathrm{~cm}$ clear Plexiglas panel that serves as a response manipulandum. When touched or pressed. each panel operates a microswitch (Figure 2). Fourteen centimeters directly below the center of each stimulus window is a $6 \times 7.5 \times 2 \mathrm{~cm}$ foodwell with translucent Plexiglas sides that can be illuminated.

Operation of any one of the three response manipulanda triggers the response circuitry. electrically locks out subsequent effective responses. and, if the response is correct, activates a solenoid reward mechanism which has been preloaded by the experimenter (see Figure 2). An effective response also results in illumination of the foodwell located betow the manipulandum responded to, independent of reward.

Located behind the stimulus windows are specially constructed Plexiglas cartridges that hold closed loops of $70-\mathrm{mm}$ perforated photographic film. A single film loop is $44 \mathrm{~cm}$ in circumference. Each cartridge (see Figure 2) has an aluminum roller fitted to a $0.6-\mathrm{cm}$ 


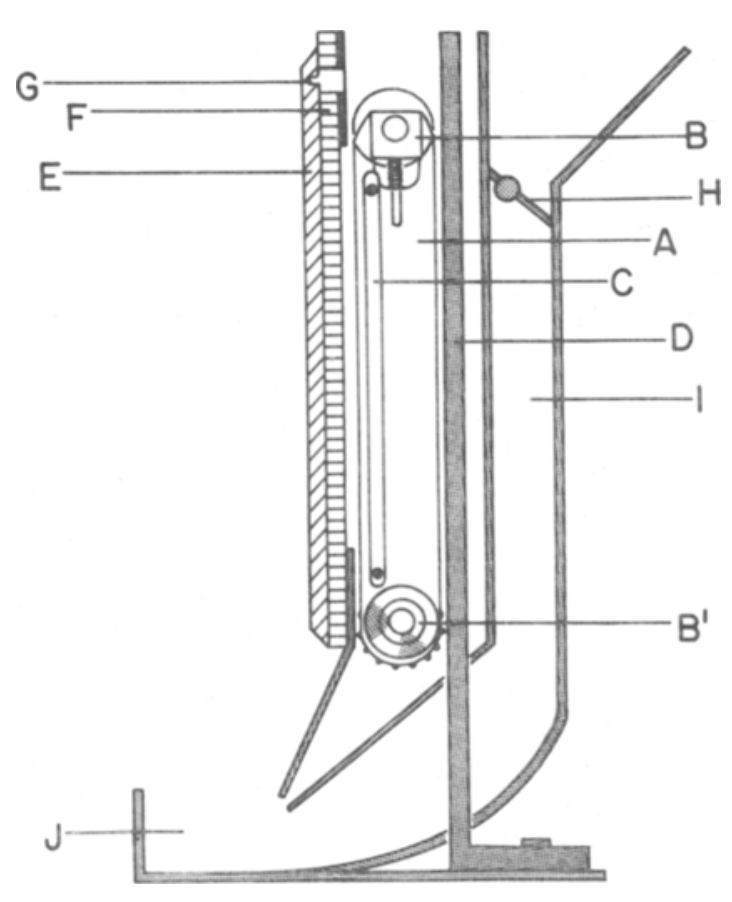

Figure 2. Side view of a film-loop cartridge (A) shows the idler roller $(B)$ and drive sprockets $\left(B^{\prime}\right)$ around which the closed loop is driven so that the stimulus passes in front of the rear-projective surface $(C)$. The cartridge is held in position by upright tracks $(D)$ so that the drive-shaft pressure roller rests on a similar roller attached to the motor-drive mechanism (not shown). When the clear Plexiglas manipulandum $(E)$ that overlays the stimulus window (F) is pressed, a microswitch $(G)$ is activated. On correct trials, a solenoid-controlled reward gate $(H)$ opens, thereby allowing the reward to pass through the delivery chute (I) to the foodwell (J).

steel shaft that rotates in spring-loaded brass bushings, keeping the film loop under sufticient tension to prevent buckling or warping as it is driven around the idler roller at the top of each cartridge, and $35-\mathrm{mm}$ nylon sprockets located at the cartridge base. These drive sprockets are fitted to a steel shaft that rides in brass bushings. Affixed to one end of the shaft is a rubber pressure roller that comes into contact with a similar roller mounted on a reversible motor-drive mechanism once the cartridge has been slid into positioning holders.

When a cartridge is seated in one of the three holders. the tilm surface is in a vertical plane parallel to the corresponding stimulus window. Each side of the cartridge is situated directly in front of a bank of six Chicago Miniature No. 1251 lamps. Since the cartridge sides are clear Plexiglas, light from these lamps diffuses through a frosted Plexiglas divider located directly behind the front surface of the film. Each lamp operates at $6 \mathrm{~W}$, thereby providing a total of $36 \mathrm{~W}$ of illumination for each film-loop cartridge. Two-dimensional objects or repetitive patterns can be reverse-contact printed on the film strips, leaving a clear stimulus on the black exposed portion of the film. Consequently, the resultant pattern is rear-illuminated and appears as a brightly colored stimulus to the subject. Specific colors can be obtained by changing the lamps or by coloring the tilm loops when white light is employed.

Stimuli may be given vertical motion, either upward or downward, by activating the motor drive unit upon which each cartridge rests. A cartridge indexing feature repositions the film loop to a predetermined starting position following completion of a trial, thereby insuring a uniform three-stimulus spatial configuration among the three stimulus windows. Motion is initiated by activating a clutch (Hurst 60-rpm PC-DA motors) which allows synchronous movement of two or three stimuli. Film speed is approximately $8 \mathrm{~cm} / \mathrm{sec}$, so that a single stimulus of $4 \mathrm{~cm}$ height traverses the vertical distance of a stimulus window in approximately $1.75 \mathrm{sec}$. If two stimuli are spaced equidistant from each other on a film loop, the second image comes into view just as the lirst image disappears.

The stimulus display console is connected via a 24-conductor cable to a control console. This unit and the experimenter are hidden from the subject's view by the display console when its cover plate is rotated into an upright position (see Figure 1). The control console contains all the necessary electrical components and control devices to operate the entire display unit.

\section{OPERATION}

The desired stimulus contigurations for the three uindows can be accomplished in two ways: (1) manually interchanging the film cartridges to obtain the appropriate position order, or (2) advancing and indexing each film loop to the appropriate stimulus via the control console switches. The tester's procedure is then: (1) select desired movement, (2) select stimulus color, (3) choose correct choice stimulus, (4) index, (5) start trial, (6) raise forward screen. (7) following subject's response, lower screen, (8) stop trial, and (9) reload reward magazine.

Experienced rhesus monkeys respond quite rapidly in this test situation, usually well within a $2-\mathrm{sec}$ period so that an experienced tester can comfortably adninister a single trial within $7-10 \mathrm{sec}$. To assess the facility with which rhesus monkeys solve simple learning problems administered with this apparatus, we conducted a pilot study involving three-choice discrimination tasks. ${ }^{1}$

\section{EXPERIMENT}

\section{Method}

Subjects. Three 6-year-old WGTA-sophisticated thesus monkeys 
(Macaca mulatta) served as subjects. Although they had not previously solved three-choice discrimination problems.' each subject had mastered two-choice object discriminations and two-choice oddity problems by age 5 years. The monkeys had not been WGTA tested for 1 year prior to the start of the present experiment and none had received any previous testing with two-dinensional stimuli.

Procedure. The monkeys were trained to panel-press under $100 \%$ CRF. Initially, the transparent response panels were baited with small bits of Marshmallow until the subject's response consistently activated the microswitch controlling reward delivery. Consistent panel-pressing to unbaited stimulus windows developed rapidly. requiring an average of approximately 25 baited trials. The monkeys were further trained for 2 100-trial days on a simple discrimination problem involving a single red or green triangle $(100 \% \mathrm{CRF})$ which appeared at random in one of the three stimulus windows. the other windows remaining opaque black. The mean correct response level for the three subjects and two sessions was $74 \%$.

Four three-choice discrimination problems were then administered in random order in 100-trial blocks per day until a criterion of 9 out of 10 correct responses was reached by the noncorrection method. In Problem C. a green triangle vs. two red triangles was presented. Problem $M$ consisted of three green triangles, one of which was in downward motion and designated the correct choice. Problems CR and MR were reversal problems, i.e., in $C R$, a red square vs. two green squares was rewarded, and in $M R$, a static red square vs. two dynamic red squares was rewarded.

\section{Results and Discussion}

The mean number of trials to criterion for Color Problems $C$ and $C R$ across the three subjects was 82 ; the same statistic for Motion Problems $M$ and $M R$ was 66 . An average of 101 trials were required to reach criterion on the $C$ and $M$ problems combined, while the mean number of trials to criterion for the reversal problems, $C R$ and $M R$, was 47.
It appears that adequate performance levels can be obtained with the present apparatus, although its general application to studies of primate learning remains to be thoroughly tested. The apparatus is sufficiently flexible to permit numerous and varied applications. Additionally, it seems that subjects are more likely to touch the relevant cues in responding to two-dimensional stimuli presented in this way, rather than the usual WGTA procedure which involves patterns printed on cards.

\section{REFERENCES}

Hamilton, C. R., \& Lund, J. S. Visual discrimination of movement: Midbrain or forebrain? Science, 1970, 170, 1428-1430.

Harlow, H. F. The formation of learning sets. Psychological Review, 1949, 56, 51-65.

Sayner, R. B., \& Davis, R. T. Significance of sign in an S-R separation problem. Perceptual and Motor Skills, 1972, 34. $671-676$.

Spaet, T., \& Harlow, H. F. Solution by rhesus monkeys of multiple sign problems utilizing the oddity technique. Journal of Comparative Psychology, 1943, 35, 119-132.

WARREN, J. M. Solution of sign-differentiated object and positional discriminations by rhesus monkeys. Journal of Genetic Psychology, 1960, 96, 365.

\section{NOTE}

1. A simple oddity problem which does not include the oddity principle is sometimes called one-odd oddity since there is no intertrial stimulus reversal, i.e., the subject can adopt a strategy of responding to the same stimulus from trial to trial.

(Received for publication October 24, 1974; revision received December $21,1974$. 